"Demokratie"-Ansprüchen genügen sollen. Diesem Thema kann sich auch vom Grundsätzlichen her nähern, wer bei Stefan Brüne / Oskar von Maltzan ("Demokratische Transition: Wahlen und Wahlbeobachtung in Guinea", Jahrbuch 1996; entsprechend für Südafrika schon Theodor Hanf im Jahrbuch 1995) einsetzt oder beim Autorenteam Helga Fleischhacker / Michael Krennerich / Bernhard Thibaut ("Demokratie und Wahlen in Afrika und Lateinamerika: Eine Bilanz der neunziger Jahre", Jahrbuch 1997). Die hier erstellte tabellarische Klassifizierung nach - vor allem - der Freiheit und Fairness von Wahlen weist aus, daß die "Regime im Übergang" $55 \%$ aller afrikanischen Staaten ausmachen, während bei den "Demokratien" Lateinamerika mit $70 \%$ seiner Staaten vorn liegt.

Last not least finden ökonomische Themen ausreichend Platz. Fragen interregionaler Kooperation behandeln für die amerikanische Hemisphäre Barbara Fritz (zum MERCOSUR, Jahrbuch 1995) und Detlef Nolte (zur "Cumbre de las Américas", Jahrbuch 1996), für den Mittleren Osten Aziz Alkazaz (zur "Economic Cooperative Organisation ECO", Jahrbuch 1996) und zum asiatisch-pazifischen Raum (ASEAN/APEC) Klaus-Albrecht Pretzell ("Die APEC auf dem Weg zur Institutionalisierung", Jahrbuch 1995). Im gleichen Band ein "Muß" zum Verständnis der dortigen Wirtschaftsabläufe: die faszinierende Studie von Rüdiger Machetzki zur Wirkungsweise überseechinesischer Familien- bzw. "Guanxi"Unternehmen ("Kultur und Wirtschaftserfolg in Südostasien"). Neueste Entwicklungen im Verhältnis EU/Lateinamerika beschreibt Detlef Nolte ("Die Wiederentdeckung Lateinamerikas durch Europa", Jahrbuch 1997) im wesentlichen vom Handelspolitischen her (MERCOSUR, NAFTA, FTAA, Andenpakt), läßt aber auch weitergehende Aspekte deutlich hervortreten, vor allem die Re-Animation auch der deutschen Lateinamerika-Politik.

Bei dieser Themenfülle bleibt die Eindringtiefe einer Rezension begrenzt. Immerhin sollte der nach wie vor imponierende Informations-Fundus dieser Reihe deutlich geworden sein. Vor 14 Jahren angetreten, um "... mit jedem Band zu einem umfassenden, jeweils auf dem neuesten Stand befindlichen Handbuch der Dritten Welt zu werden ...", wird diese Reihe auch künftig keine Wünsche offen lassen - und dies zu einem für jedermann erschwinglichen Preis.

Karl-Andreas Hernekamp

\title{
Strafgesetze der Islamischen Republik Iran
}

Übersetzt und eingeleitet von Silvia Tellenbach.

Sammlung außerdeutscher Strafgesetzbücher in deutscher Übersetzung, hrsg. vom MaxPlanck-Institut für ausländisches und internationales Strafrecht in Freiburg i. Br., CVI, de Gruyter Verlag, Berlin, 1996, 190 S., DM 116,--

In den intensiv und voller Emotionen geführten Diskussionen um die Islamisierung von Gesetzen in den Ländern der islamischen Welt steht der Bereich des Straf rechts nicht von unge- 
fähr im Mittelpunkt. Die strafrechtlichen Normen widerspiegeln augenfällig die historischen Leistungen und gegenwärtigen Probleme des islamischen Rechts. Für den mit der arabischen oder einer anderen orientalischen Sprache wenig vertrauten Leser mangelt es an verläßlichen Textquellen zur Beurteilung des islamisierten Strafrechts (abgesehen von einigen Veröffentlichungen zum Sudan, O. Köndgen). Die strafrechtlichen Veränderungen nach dem Sieg der Islamischen Revolution in Iran waren für den deutschsprachigen Leser lediglich partiell durch die 1984 im Kölner Media Pro Verlag erschienene Übersetzung des "Gesetzes über hudûd und qisâs" (D. Ilzad) zu erfassen. Daher ist es um so erfreulicher, daß sich die Autorin als ausgewiesene Spezialistin des orientalischen Rechts und der orientalischen Philologie der ebenso mühsamen wie schwierigen Aufgabe stellt, das im Jahre 1991 angenommene "Gesetz über die islamischen Strafen", welches auf drei entsprechenden Gesetzen aus dem Jahre 1982 basiert, sowie das seit 1983 gültige "Gesetz über die ta'zîrât", welches nunmehr auch seine parlamentarische Bestätigung erhielt, zu übersetzen und in einer knappen, aber instruktiven Einleitung zu kommentieren (S. 1-24). Die zahlreichen Nebengesetze, auf die Tellenbach aufmerksam macht (S. 24) und die in einer persischen Sammlung von relevanten Rechtsakten aus dem Jahr 1986/87 auf mehreren hundert Seiten erfaßt sind, konnten bis auf zwei Gesetze im Anhang (S. 183-190) verständlicherweise nicht berücksichtigt werden.

Das vorliegende Buch überzeugt nicht nur durch eine stilistisch hervorragende Übersetzung, sondern auch durch erläuternde Anmerkungen, die für das Verständnis des Textes unabdingbar sind. Die arabische Umschrift (auch für persische Wörter) - für den Laien eher verwirrend - wird erfreulicherweise nur sparsam verwendet. Einige kleinere diesbezügliche Druckfehler (S.4: pâsuh; S. 16: qat ${ }^{\mathrm{c}}$ ) fallen daher kaum ins Gewicht.

Die Autorin erläutert zunächst die neuere Geschichte des Straf rechts in Iran und verweist auf die Zäsur der Islamischen Revolution (S. 1-5). Der kurzen Darstellung des islamischen Strafrechts (unter Hinweis auf die Ausführungen von A. El Baradie) folgt eine sehr ausgewogene Einführung in das Gesetz aus dem Jahre 1991 mit seinen vier Büchern (Allgemeines, h addStrafen, Vergeltung - qisâs, Blutgeld - diya). Ohne störende Polemik arbeitet Tellenbach die Spezifika des schiitisch geprägten Straf rechts heraus und unterstreicht, daß die Systematik des Gesetzes nur bedingt mit der Struktur eines "westlichen" Strafgesetzbuches verglichen werden kann (S. 8). Wie sich islamische Rechtsfiguren unter veränderten Bedingungen anwendbar machẹn lassen, demonstriert das in den Art. 183-196 formulierte Delikt des "Kampfes gegen Gott und Verderbenstiften auf Erden" (S. 16-17 und 66-69). Der Hauptanwendungsbereich dieser Tatbestände liegt in politischen Straftaten (S. 17). Hier muß für Iran eine traurige und blutige Bilanz gezogen werden. Tötung und Körperverletzung ziehen im Falle des Vorsatzes Vergeltung nach sich. Dagegen wird die quasi-vorsätzliche (oder auch vorsatzähnliche) und fahrlässige Tat grundsätzlich mit Blutgeld gemäß Art. 294-497, Viertes Buch; S. 95-138) bestraft. Art. 206 definiert die vorsätzliche Tötung, Art. 271 analog die vorsätzliche Körperverletzung. Der Gesetzgeber spricht im Art. 295b von einer "vorsatzähnlichen Fahrlässigkeit", die neben der fahrlässigen Tat und bei Unzulässigkeit der Vergeltung Blutgeld erfordert.

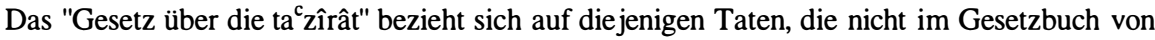
1991 erfaßt sind. Tellenbach hebt in der Einleitung die im Vergleich zum Besonderen Teil des 
Strafgesetzbuches von 1926 erfolgte Änderung des Strafensystems hervor. Vierundsiebzig Peitschenhiebe werden als häufige Strafe verankert (S. 23), im übrigen auch für Frauen, die sich in der Öffentlichkeit ohne vorschriftsmäßige Kleidung (d. h. unverschleiert) zeigen (Art. 102).

Ein Blick in die Strafgesetze der Islamischen Republik Iran, den die Autorin durch ihre Veröffentlichung in deutscher Sprache ermöglicht, sollte nicht nur für den Juristen und Strafrechtler, sondern auch für denjenigen von Interesse sein, der sich mit Religion und Gesellschaft Irans beschäftigt. Ihr gebührt der Dank für eine rundum gelungene Arbeit. Der Leser darf auf ihre zukünftigen Veröffentlichungen gespannt sein.

Hans-Georg Ebert

\section{Florian Amereller}

Hintergründe des "Islamic Banking". Rechtliche Problematik des riba-Verbotes in der Sharia'a und seine Auswirkungen auf einzelne Rechtsordnungen arabischer Staaten Schriften zum Internationalen Recht, Bd. 71

Duncker \& Humblot Verlag, Berlin, 1995, 206 S., DM 112,--

Die Rolle des islamischen Rechts ist in Handelsbeziehungen mit arabischen Ländern, vor allem mit den Golfstaaten, ein großer Unsicherheitsfaktor. Viele international übliche Vertragsklauseln können vor dem klassischen islamischen Recht nicht bestehen. Stolpersteine bilden vor allem das Verbot von gharar, was etwa mit "Unsicherheit" oder "Unbestimmtheit" übersetzt werden kann, und von riba, was gemeinhin unscharf im Sinne von "Zins" oder "Wucher" verstanden wird. Die Staaten der islamischen Welt stehen vor dem Problem, religiös fundierte Normen mit den Anforderungen des heutigen Wirtschaftslebens zu versöhnen. Das Problem wird noch dadurch verschärft, daß die Aussagen des klassischen islamischen Rechts gerade in diesen Bereichen alles andere als eindeutig sind. Ihre zentrale Stellung im Islam macht sie jedoch zu einem Kristallisationspunkt von Reislamisierungsbestrebungen, die immer auch mit einer Abwehr tatsächlicher oder vermeintlicher Überf remdung einhergehen.

Amerellers Arbeit, eine Mainzer juristische Dissertation, hat sich zum Ziel gesetzt, das riba-Verbot darzustellen und seinen Einfluß auf das heutige Zivilrecht arabischer Staaten zu untersuchen. Ein solches Vorhaben setzt nicht nur juristische Vorbildung, sondern auch erhebliche Kenntnisse in Sprache und Kultur voraus und ist schon deswegen anerkennenswert. Durch den aktuellen Bezug ist sie darüber hinaus auch praxisrelevant und verspricht Aufschlüsse über das Kräfteverhältnis von, grob gesagt, Islamisierungs- und Säkularisie- 